International Journal of Computing and Business Research (IJCBR)

ISSN (Online) : 2229-6166

International Manuscript ID : 22296166V7|1201706

Volume 7 Issue 1 May - June 2017

\title{
Trajectory Data Warehouse and Design Perspectives
}

\author{
Halgare Nanasaheb Mahadev \\ Research Scholar \\ Kalinga University, Chhattisgarh, India \\ Dr. Ali Akbar Bagwan \\ Research Supervisor \\ Kalinga University, Chhattisgarh, India
}

and/or an expense streams among arrangements of better places in a town (inception destination streams) is a key issue.

\begin{abstract}
KEYWORDS: Trajectory Data Warehouse, Applications and Domains of Trajectory Data Warehouse, Satellite Based Applications

Spatio-worldly examples that compactly demonstrate the aggregate conduct of a populace of moving items are valuable deliberations to comprehend portability related wonders. Specifically, a type of example, which speaks to an accumulated deliberation of numerous individual directions of moving articles inside a watched populace, would be to a great degree valuable in the space of reasonable portability and movement administration in metropolitan zones, where the revelation of activity Permission to make advanced or printed copies of all or some portion of this work for individual or classroom use is conceded without charge gave that duplicates are not made or conveyed for benefit or business advantage and that duplicates bear this notification and the full reference on the principal page. To duplicate generally, to republish, to present on servers or on redistribute to records, requires earlier particular authorization
\end{abstract}

These days, the development of individuals or vehicles inside a given range can be seen from the computerized follows left behind by the individual or vehicular cell phones, and gathered by the remote system frameworks. Case in point, cellular telephones leave situating logs, which indicate their confinement, or cell, at every minute they are associated with the GSM system; comparably, GPSprepared versatile gadgets can record their scope longitude position at every minute they are presented to a GPS satellite, and transmit their directions to a gathering server. The pervasiveness of universal innovations ensures that there was be an expanding accessibility of a lot of data relating to individual directions, at expanding limitation exactness; in this manner, there is an open door - and a test - to find naturally, from these directions, spatio-fleeting examples that pass on helpful learning.

In this study, we unequivocally address this issue, and present a novel type of spatio-worldly example, which formalizes the specified thought of total 
International Journal of Computing and Business Research (IJCBR)

ISSN (Online) : 2229-6166

International Manuscript ID : 22296166V7I1201706

Volume 7 Issue 1 May - June 2017

development conduct. The new example, that we call a direction design, speaks to an arrangement of individual directions that share the property of going to the same succession of spots with comparable travel times. Hence, two ideas are focal:

(i) The districts of enthusiasm for the given space, and

(ii) The run of the mill travel time of moving items from locale to area.

Lately Global Positioning Systems (GPS) have turned out to be progressively accessible and exact in cell phones. Accordingly a lot of spatio-temporal reality and figures are being produced by clients of such cell phones, alluded to as moving items in the accompanying. Directions of moving items, or directions for short, contain regularities or examples. For instance, a man tends to drive verging on each weekday to work roughly in the meantime utilizing the same course.

The advantages of discovering such regularities or examples are many-fold. Initially, such examples can help the productive Proceedings of the third Workshop on STDBM Seoul, Korea, September 11, 2006 administration of directions. Second, they can be utilized to encourage different Location-Based Services (LBS). One LBS illustration is an astute rideshare application, which finds sharable courses for an arrangement of workers and recommends rideshare conceivable outcomes to them, is viewed as; such rideshare application can be one conceivable answer for the perpetually expanding blockage issues of urban transportation systems.

Designs in directions for an insightful rideshare application are just fascinating if those examples are sharable by different workers, are reoccurring regularly, and are beneficial seeking after, i.e., are sufficiently long for the funds to make up for the coordination endeavors. The disclosure of Long, Sharable Patterns (LSP) in directions is troublesome for a few reasons.
Examples don't more often than not exist along the entire direction. As an illustration, consider two suburbanites An and B living in the same neighborhood, leaving for work around the same time, and working in the same piece of town. Given the fundamental street system and activity conditions, for a given bolster limit the center part of the treks of the two workers might be regular; the underlying and last parts may not. In late work a general issue change strategy, called turning, was proposed for the examination of spatio-temporal data. Turning is the system of collection an arrangement of records in view of an arrangement of characteristics and doling out the estimations of likely another arrangement of ascribes to gatherings or wicker container. Turning connected to spatio-temporal data permits the development of spatio-temporal wicker container, which can be mined with customary affiliation principle mining calculations. At the point when the things in the wicker container are spatio-temporal identifiers and are gotten from directions, the found principles speak to oftentimes voyaged courses, while there exist a few effective affiliation standard mining techniques, the straight-forward utilization of these calculations to spatio-temporal crate speaking to directions is infeasible for two reasons. To start with, all sub-patterns of incessant examples are likewise visit, yet not intriguing, as longer examples are favored. Second, the bolster foundation utilized as a part of affiliation guideline digging calculations is deficient for a rideshare application, i.e., a continuous thing set speaking to a successive direction example, might be upheld by a solitary suburbanite on numerous events and thus displays no rideshare opportunity.

Directions have been for the most part considered as the way took after by an item moving in space and time (Wolfson et al. 1998, Guting et al. 2006). Every point in this way speaks to one position in space and one moment in time. Commonly, direction data are acquired from cell phones that catch the position of an item at particular time interims. The foundation geographic data on which articles are moving is not caught by these gadgets, but rather is of central significance for the examination of direction data in

\section{Approved with the Standing Committee of UGC (University Grants Commission), Govt. of India UGC Approved List Journal No. 45420}




\section{International Journal of Computing and Business Research (IJCBR)}

ISSN (Online) : 2229-6166

International Manuscript ID : 22296166V7I1201706

Volume 7 Issue 1 May - June 2017

genuine applications. In this manner, there is an expanding need for a more important representation of directions, and also their organization with the geographic space. We can envision an arrangement of directions, from which very little data can be removed.

Trajectory pattern mining

We are seeing a quick and preceded with dissemination of cell phones, for example, PDAs, individual route gadgets and tablet PCs. Further, these gadgets are progressively being geo-situated utilizing satellite route frameworks, e.g., GPS, frameworks that endeavor the remote correspondence base, and closeness based frameworks, e.g., RFIDbased frameworks.

The subsequent area gadgets find boundless use in different business and individual settings in the public eye. As a result of this extension, expanding volumes of position data are being collected, and the capacity of examining substantial volumes of direction reality and figures is in expanding request in a wide range of uses. Critical applications in different areas need to distinguish and use gatherings of directions that show comparable examples from an accumulation of directions. Case applications incorporate transportation advancement, expectation empowered administrations, logical and social examination applications, sports investigations, and additionally group and exception examinations.

Transportation streamlining applications need to discover gatherings of comparative directions that show that the relating objects voyaged together. Case in point, an auto pooling application may interface drivers in the same direction bunch keeping in mind the end goal to lessen their travel costs. A logistics application may look at the conveyance trucks in the same gathering keeping in mind the end goal to accomplish better arranging.

Prediction strategies may misuse information of direction gatherings for the comprehension of item conduct. Such learning can be utilized for offering viable warnings, for the conveyance of promotions to focused group of onlookers, and for giving redid area based administrations.

Scientific studies may require the distinguishing proof of gatherings of creatures that moved together. They are valuable in finding creature development designs (e.g., honey bees, an assortment of winged creatures, ocean turtles, whales, and fish), in discovering crowds of creatures, and in concentrating on creature conduct designs in natural surroundings. So also, social examination studies may intend to recognize financial examples from run of the mill development examples of people.

Team sports occasions (soccer, baseball, hockey, rugby, computerized war zones) likewise give significant direction certainty and assumes that catch the players' developments. By concentrating on a diversion as gatherings of directions, it might be conceivable to better comprehend the amusement, to break down the strategies utilized as a part of the diversion, and to try and concentrate the area and time of utilizing a specific technique.

Traffic examination applications may use direction bunches for the investigation of group and anomalies. In this situation, a moving article can be either vehicles out and about or a person on foot in the city. A huge direction gathering is prone to demonstrate a group conduct. By recognizing swarms from the directions, a superior comprehension of group is conceivable, e.g., the times and places when and where swarms shape and break down. Such data might be abused for overseeing transportation foundations viably.

It is additionally important to mine exceptions, which don't have a place with any direction bunch. This might be utilized for identifying and evacuating mistakes in the direction reality and figures (e.g., finding a gadget with a breaking down GPS beneficiary). It might likewise be connected for recognizing perilous driving practices. 


\section{International Journal of Computing and Business Research (IJCBR)}

ISSN (Online) : 2229-6166

International Manuscript ID : 22296166V7I1201706

Volume 7 Issue 1 May - June 2017

Direction design mining is a rising and quickly creating point in the territories of reality and figures mining and question administration that goes for finding gatherings of directions in view of their vicinity in either a spatial or a spatiotemporal sense. The writing contains an assortment of late proposition around there.

Existing recommendations speak to various exchange offs in the expressiveness of the direction designs concentrated on. Considering just confined examples may bring about not having the capacity to distinguish fascinating marvels from the reality and figures, though considering entirely casual examples may prompt the reporting of irrelevant examples while, existing proposition likewise accompany their own file structures and mining calculations that intend to empower proficient and versatile revelation of examples in expansive direction data sets. This study displays an outline of the key ideas and disclosure procedures in best in class concentrates on in the mining of direction examples.

\section{Global Vs. Fractional Patterns}

Direction examples can be ordered in light of the fleeting degree of the directions that take part in the examples. In worldwide direction designs, directions are seen as non-decomposable, i.e., the essential unit of example disclosure is an entire direction.

For instance, Gaffney et al propose a model-based bunching calculation that gatherings directions with general comparative courses utilizing a relapse blend model and the EM calculation. This calculation creates bunches of directions concerning the general separations among entire directions.

Conversely, fractional direction designs consider incomplete directions in the example disclosure method. The thought behind this methodology is that the bunching of entire directions may not distinguish directions with comparable sub-directions. When all is said in done, a direction might be long and complex, Henceforth, despite the fact that a few sections of directions are comparable; the full directions won't be comparative situated in this perspective, propose to segment directions into line sections and to assemble gatherings of close direction portions.

Singular Vs. Bunch Patterns - Another qualification identifies with whether an arrangement of individual directions are recovered that fulfill an example determined in question, called singular direction design recovery, or whether sets of directions are recovered so that the directions in a set display a comparative example as indicated by some particular idea of example, called bunch direction design revelation So-called spatiotemporal example inquiries delineate well the recovery of directions that fulfill a specific example as determined in an inquiry. Case of such questions incorporate the finding of flights that slipped into an airplane terminal, however did not arrive; recognizing flights that needed to make a few methodologies before entering the air terminal; and finding vessels that changed course to dodge a crash.

\section{Conclusion:}

Our methodology more or less, As directions stream we segment the approaching stream in windows, every window being divided in slides, with a specific end goal to diminish the extent of the info directions we pre-technique every approaching direction keeping in mind the end goal to get a littler representation of it as a grouping of locales. We call attention to that this operation is appropriate in our system since we are not keen on point-level developments but rather in directions shapes. The rejoining methodology we abuse utilizes PCA to better distinguish headings along which we ought to play out a more exact parcel slighting areas not on the key bearings. The method of reasoning for this presumption is that we look for regular directions so it is improbable that areas far from chief headings was add to continuous examples (in the accompanying we was use successive examples and incessant directions as equivalent word). The arrangement of areas so far got can be spoken to as a 


\section{International Journal of Computing and Business Research (IJCBR) \\ ISSN (Online) : 2229-6166 \\ International Manuscript ID : 22296166V7|1201706 \\ Volume 7 Issue 1 May - June 2017}

string for which we can abuse a reasonable form of understood regular string mining calculations that works effectively both regarding space and time utilization.

\section{REFERENCES}

[1] Leonardi, L., Marketos, G., Frentzos, E., Giatrakos, N., Orlando, S., Pelekis, N., ... \& Theodoridis, Y. (2010, March). Twarehouse: visual olap analysis on trajectory data. In Data Engineering (ICDE), 2010 IEEE 26th International Conference on (pp. 1141-1144). IEEE.

[2] Wagner, R., de Macedo, J. A. F., Raffaetà, A., Renso, C., Roncato, A., \& Trasarti, R. (2013, November). Mob-warehouse: A semantic approach for mobility analysis with a trajectory data warehouse. In International Conference on Conceptual Modeling (pp. 127-136). Springer, Cham.

[3] Orlando, S., Orsini, R., Raffaetà, A., Roncato, A., \& Silvestri, C. (2007). Trajectory data warehouses: Design and implementation issues. Journal of computing science and engineering, 1(2), 211-232.

[4] Campora, S., de Macedo, J. A. F., \& Spinsanti, L. (2011). St-toolkit: A framework for trajectory data warehousing. In 14th AGILE Conference on Geographic Information Science. Utrecht, Holanda.

[5] Arfaoui, N., \& Akaichi, J. (2011). Modeling herd trajectory data warehouse. International Journal of Engineering Trends and Technology, 6, 1-9. 